\title{
The Electronic Structure of Amorphous Carbon
}

\section{Nanodots}

Johannes T. Margraf, ${ }^{1}$ Volker Strauss, ${ }^{2}$ Dirk M. Guldi, ${ }^{2 *}$ Timothy Clark ${ }^{1,3, *}$

${ }^{1}$ Computer-Chemie-Centrum and Interdisciplinary Center for Molecular Materials, Department Chemie und Pharmazie, Friedrich-Alexander-Universität Erlangen-Nürnberg, 91052 Erlangen, Germany, ${ }^{2}$ Department of Chemistry and Pharmacy and Interdisciplinary Center for Molecular Materials, Friedrich-Alexander-Universität Erlangen-Nürnberg, 91058 Erlangen, Germany and

${ }^{3}$ Centre for Molecular Design, University of Portsmouth, Portsmouth PO1 2DY, United Kingdom.

Abstract: We have studied hydrogen-passivated amorphous carbon nanostructures with semiempirical molecular orbital theory in order to provide an understanding of the factors that affect their electronic properties. Amorphous structures were first constructed using periodic calculations in a melt/quench protocol. Pure periodic amorphous carbon structures and their counterparts doped with nitrogen and/or oxygen feature large electronic band gaps. Surprisingly, descriptors such as the elemental composition and the number of $\mathrm{sp}^{3}-$ atoms only influence the electronic structure weakly. Instead, the exact topology of the $\mathrm{sp}^{2}$ network in terms of effective conjugation defines the band gap. Amorphous carbon nanodots of different structures and sizes were cut out of the periodic structures. Our 
calculations predict the occurrence of localized electronic surface states, which give rise to interesting effects such as amphoteric reactivity and predicted optical band gaps in the nearUV/visible range. Optical and electronic gaps display a similar dependence on particle size to inorganic colloidal quantum dots.

KEYWORDS: semiempirical molecular orbital theory; nanoparticles; quantum dots; periodic boundary conditions

\section{Introduction}

In recent years, many examples of luminescent, colloidal, carbon-based materials, which have been synthesized in solution, have been reported under the umbrella term "carbon nanodots" (CNDs). ${ }^{1}$ It has been suggested, and is widely accepted that graphene-like domains of $\mathrm{sp}^{2}$ carbons in conjunction with trap states are mainly responsible for the corresponding photophysical properties. ${ }^{2}$ In many cases, reports regarding carbon nanodots provide experimental evidence of the formation of graphitic or aromatic structures. The assignment of the major optical features of carbon nanodots to planar, aromatic carbon domains is inherently reasonable but structural characterization is often ambiguous because "dark" features such as non-conjugated linkages or structures cannot be observed. No consistent structural or electronic picture of CNDs is available other than that they consist of graphene-like moieties. Such features may indeed be present in amorphous CNDs but not exclusively. This is underlined by the strong dependence of the photophysical properties of CNDs on the method of preparation. ${ }^{3}$ We therefore now report semiempirical molecular-orbital (MO) calculations on models for amorphous CNDs that do not assume graphenic structures from the outset in order to gain insight into the likely structures and electronic properties of CNDs. It is important to consider such models in order to understand the 
structural features and electronic characteristics of CNDs systematically, so that they can be tailored rationally in order to obtain the desired properties for electronic applications.

All theoretical models of CNDs of which we are aware are based on graphene nanoflakes. ${ }^{4-13}$ Given that amorphous carbon of low density also contains a high ratio of $\mathrm{sp}^{2}$-carbon atoms, we probed the potential electronic properties of carbon nanodots with amorphous-like structures. In particular, we were interested in materials that might emerge from low temperature (400-500 K), solventbased synthetic procedures, such as that used in our laboratory. ${ }^{3}$ Two independent theoretical studies have shown that amorphous carbon nanoparticles with low or no hydrogen-content might even be (semi-)metallic. ${ }^{14,15}$ In stark contrast, we consider hydrogen passivated structures that feature large band gaps because these are better models for the real situation, in which nonfunctionalized carbon surfaces and edges are unlikely.

The mechanism of the low-temperature synthesis of carbon nanodots that starts from molecular precursors is not well understood. It is assumed that, following an initial polymerization of the precursors, the polymers coil into dense aggregates. In a final step, these coils are carbonized, with heteroatom-based leaving-groups being expelled. ${ }^{16}$ We designed our theoretical approach to mimic this mechanism.

We have studied amorphous carbon nanostructures with neglect of diatomic differential overlap (NDDO) semiempirical molecular-orbital theory, as implemented in EMPIRE13. ${ }^{17}$ We have chosen semiempirical MO theory for several reasons. Firstly, the EMPIRE program is able to calculate nanostructures up to 100,000 atoms. ${ }^{17}$ However, it can also perform calculations using periodic boundary conditions, ${ }^{18,19}$ so that we can generate periodic quasi-amorphous structures and optimize their geometries. Sections of these structures can then be used for explicit cluster calculations on the nanoparticles themselves. This flexibility allows us to adopt a more realistic 
approach to the CND-structures than those reported to date, as outlined below. The periodic calculations themselves have been shown to be reliable for organic structures, ${ }^{20}$ partly because solid-state structures are often used to parameterize the Hamiltonians. ${ }^{21}$ NDDO techniques have been shown to be reliable for calculating the energies and NMR chemical shifts of fullerenes ${ }^{22}$ and to give accurate band gaps for organic semiconductors using the UHF Natural Orbital Configuration Interaction (UNO-CI) approach. ${ }^{23}$ We therefore feel confident that NDDO-theory is well suited to investigating the structural and electronic properties of CNDs, especially as the performance of the method improves for larger systems. ${ }^{22}$

Previous work has shown that liquid-quench molecular-dynamics simulations reproduce the experimental data from amorphous carbon deposited with energetic beams accurately. ${ }^{24,25} \mathrm{We}$ have therefore adapted this technique in order to build realistic quasi-amorphous CND-structures under periodic boundary conditions. Because the kinetic energy of a system synthesized in solution will be far lower than in molecular beams, we start from random structures that are quenched directly to the next energy minimum. This technique corresponds essentially to a Monte-Carlo structure generator.

The article is organized as follows: Initially, we will discuss our theoretical approach. Then, pure and heteroatom-doped amorphous carbon bulk phases are considered. Finally, results for amorphous carbon nanoparticles are shown, placing particular focus on the effects of particle size and surface states on their electronic structure.

\section{Theoretical Methods}

Amorphous bulk structures were approximated as periodic unit cells with fixed densities, each containing 128 non-hydrogen atoms. This led to unit cell sizes of approximately $10 \times 10 \times 10 \AA$, with 
slight variations depending on the chemical composition. Geometries were determined by extensive Monte-Carlo sampling. Firstly, 200-300 random geometries of a given chemical composition were generated and optimized coarsely. The ten most stable pre-optimized structures of each composition were then fully optimized. To avoid open-shell structures, whenever necessary, these geometries were saturated with hydrogen atoms and re-optimized. Typically, zero to ten hydrogen atoms were required per unit cell. Only those final geometries with closed-shell singlet ground states were used further. All 68 final structures and their corresponding data are available in the Supporting Information (Table S1 and SI_structures.zip).

The density of amorphous carbon is known to correlate with the hybridization of the constituent carbon atoms. In preliminary studies, we confirmed this dependence using our approach (see Supporting Information, Fig. S1). As known from experimental and theoretical studies, lowdensity amorphous carbons feature a higher proportion of $\mathrm{sp-}$ and $\mathrm{sp}^{2}$-carbon atoms than high density ones. This density dependence is reproduced well. Since we were interested in structures with high fractions of $\pi$-electrons, we focused on those with the relatively low density of $2.5 \mathrm{~g}$ $\mathrm{cm}^{-3}$. Unless otherwise noted, all properties are averages of all the final structures of a given chemical composition (2-8 structures).

To verify the suitability of the AM1 Hamiltonian ${ }^{26}$ for simulating periodic carbon structures, we considered diamond and graphite. Heats of formation and geometries are reproduced accurately (see Supporting Information, Figure S2) apart from the interlayer spacing in graphite. This is due to the well-known inability of AM1 to reproduce dispersion interactions. Since we are only interested in three-dimensional covalently connected amorphous networks, this should not affect the quality of our results. 
The convergence of the energy and electronic band gap within the periodic boundary conditions was tested for a $\mathrm{C}_{128}$ cell by repeating test calculations with larger supercells (see Supporting Information). These tests show that the heat of formation per atom is converged to below $0.01 \mathrm{kcal}$ $\mathrm{mol}^{-1}$ for $\mathrm{C}_{128}$. The convergence of the band gap is not as smooth and a significantly larger cell would be needed to obtain the exact value. However, the value for the $\mathrm{C}_{128}$ cell is still within 0.2 $\mathrm{eV}$ of the value for the largest unit cells tested.

Optical properties of the particles were simulated both with the semiempirical techniques AM1(UNO)-CIS ${ }^{23}$ and INDO/S $\mathrm{S}^{27}$ and with time-dependent density functional theory, using the O-LYP

functional and 6-31G(d) basis set with and without long range corrections. ${ }^{28-31}$ Because of the size of the systems, the DFT methods could only be used for the smaller dots (1-1.5 nm). TD-DFT and INDO/S calculations were performed with Gaussian $09,{ }^{32}$ UNO-CIS calculations were performed with our development version of VAMP. ${ }^{33}$

\section{Results and Discussion}

Pure Carbon: The amorphous carbon phases obtained by our approach possess an average enthalpy of atomization $\left(\Delta \mathrm{H}_{\mathrm{at}}\right)$ of $138 \mathrm{kcal} \mathrm{mol}^{-1}$. For reference, the AM1 calculated $\Delta \mathrm{H}_{\mathrm{at}}$ value of diamond is $164.4 \mathrm{kcal} \mathrm{mol}^{-1}$, while the experimental value is $171.4 \mathrm{kcal} \mathrm{mol}^{-1}$. We conclude that these structures are reasonably stable and are, in turn, realistic models of low-density amorphous carbons with low hydrogen content.

Figure 1 (left) shows a representative ball-and-stick representation for $\mathrm{sp}$ - and $\mathrm{sp}^{2}$-carbon atoms of an amorphous carbon unit cell with the $\mathrm{sp}^{3}$-framework depicted as a wireframe. Although this material features a large interconnected $\pi$-system, electron delocalization is limited by the nonplanarity of the conjugated structures. This is reflected in a relatively large electronic band gap of 
approximately $5.2 \mathrm{eV}$. Some structures also feature motifs reminiscent of polycyclic aromatic hydrocarbons (see the structure highlighted in Fig. 1, right). Although such structures are not completely $\mathrm{sp}^{2}$-hybridized, hexagonal carbon networks seem to emerge in amorphous systems. Conceptually, this establishes graphene fragments as components of carbon nanodots and thus bridges the gap between graphene and completely amorphous structures.
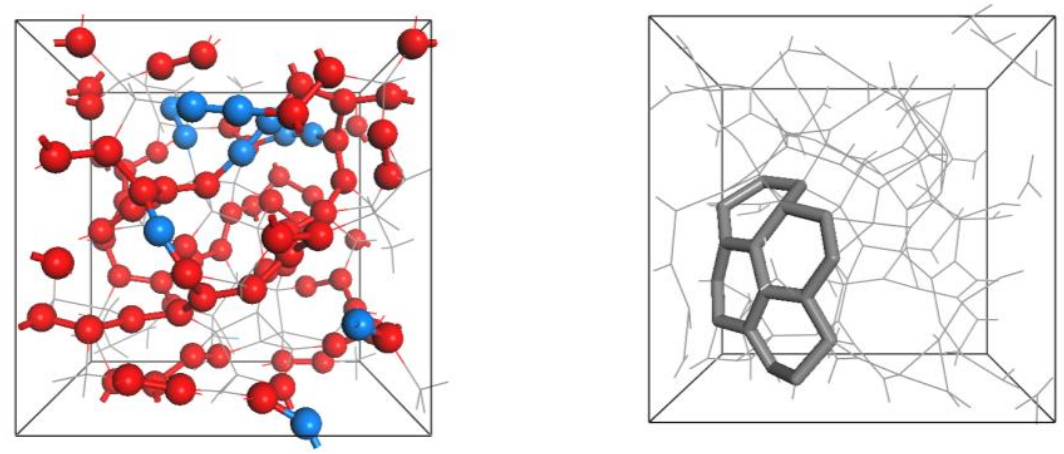

Figure 1. Left: Unit cell of a $2.5 \mathrm{~g} \mathrm{~cm}^{-3}$ amorphous carbon structure. Atoms with sp- (blue) and $\mathrm{sp}^{2}$-hybridization (red) are highlighted as balls connected with sticks. Right: A different $\mathrm{C}_{128}$ unit cell with a planar hexagonal moiety highlighted as sticks.

Heteroatoms: The influence of oxygen and nitrogen was studied by constructing a ternary phase diagram of mixed carbon, nitrogen, and oxygen phases. To this end, we varied the carbon content between 93 and 100\%, nitrogen between 0 and 4.7\% (0-6 atoms per unit cell), and oxygen between 0 and 2.3\% (0-3 atoms per unit cell). Figure 2 shows ternary phase diagrams of the average atomization energy of the most stable structures for each composition. In each case, the average atomization energy is highest for pure carbon and decreases gradually with increasing heteroatom content. 


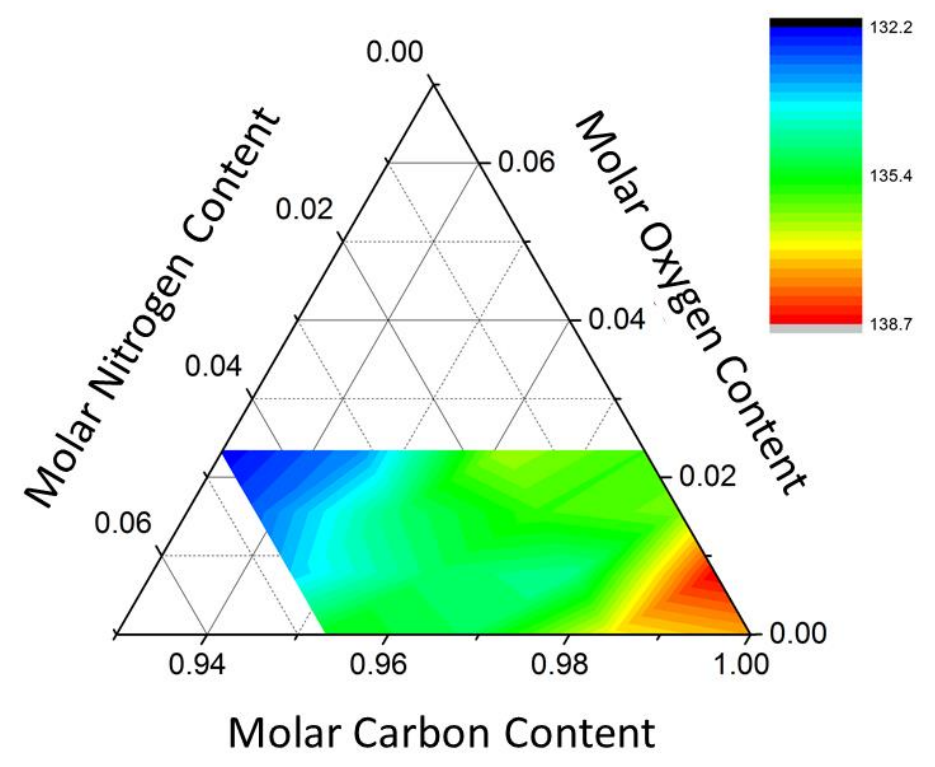

Figure 2. Ternary diagram of the atomization energy of amorphous carbon structures containing small amounts of nitrogen and oxygen (in $\mathrm{kcal} \mathrm{mol}^{-1}$ ).

The decrease in the atomization energy with increasing number of heteroatoms relates to an increase in hydrogen content for doped structures. Nonetheless, all compositions display average atomization energies within $6.5 \mathrm{kcal} \mathrm{mol}^{-1}$ of pure amorphous carbon, and are thus relatively stable.

The effect of heteroatom doping on the electronic structure is difficult to predict. The electronic band gap can be increased or decreased by the presence of heteroatoms. Small numbers of heteroatoms tend to disturb the $\mathrm{sp}^{2}$-conjugation and, in turn, to increase the band gap. In contrast, higher concentrations of either $\mathrm{N}(\sim 4 \%)$ or O $(\sim 2 \%)$ lead to decreased band gaps in some cases. In fact, the band gap variation for a group of structures with a given composition can be several eV. This is illustrated in Fig. 3 (left) for the $\mathrm{C}_{128}$ and $\mathrm{C}_{121} \mathrm{~N}_{6} \mathrm{O}_{1}$ unit cells. The elemental composition is clearly not a sufficient descriptor for predicting the electronic structure of 
amorphous carbon forms. It is therefore instructive to investigate, which structural motif causes an increase and which causes a decrease in the band gap. The strain and conjugation effects of heteroatoms on the band gaps of polycyclic aromatic hydrocarbons have been discussed in detail ${ }^{34}$ but such systematic effects are clearly not observed for the amorphous CND calculations. Experimental work on heteroatom doped amorphous carbon has been reviewed by Jones. ${ }^{35}$ Unlike crystalline semiconductors, in which doping changes the charge carrier density while the material is otherwise mostly unaffected, "doping" effects in amorphous carbon are usually connected to structural changes induced by the dopant. For example, the incorporation of oxygen has been shown to favor a clustering of six-fold aromatic rings. ${ }^{36}$ Unfortunately, the correlation length for such effects is likely above the $\sim 10 \AA$ lattice constant used in this work.
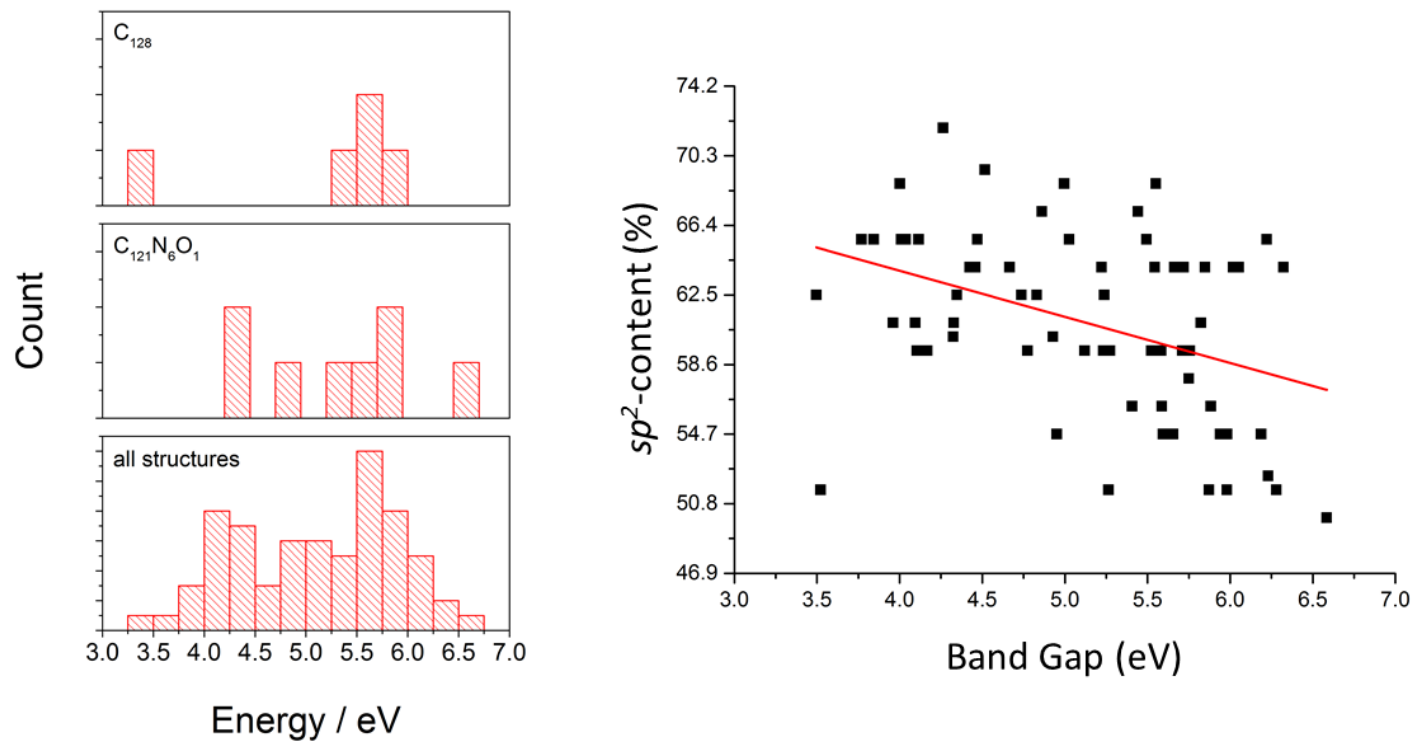

Figure 3. Left: Histograms of the electronic band gaps calculated for all structures and for $\mathrm{C}_{128}$ and $\mathrm{C}_{121} \mathrm{~N}_{6} \mathrm{O}_{1}$ unit cells. Right: Correlation $\left(\mathrm{R}^{2}=0.16\right)$ between atomic $\mathrm{sp}^{2}$-content and band gap for all amorphous structures. 
In our simulations, nitrogen atoms are mostly present in the carbon framework as amine or imine groups. In some cases, cyano groups are formed, while in one particular case molecular nitrogen is found in a pore. Oxygen atoms usually form ether or carbonyl groups. However, the expected correlation between band gap and content of $\mathrm{sp}^{2}$-hybridized atoms turns out to be barely significant (see Fig. 3, right).

The elemental composition and hybridization influence the band gap marginally, but they are not the major factors with respect to the electronic structure. Overall, the decisive factor appears to be the effective degree of conjugation, which is given by the specific topology of the structure. The determining factors are planarity, connectivity, etc. Heteroatoms only exert an influence on the electronic structure if they communicate with the $\mathrm{sp}^{2}$-network.

Since the electronic structure depends heavily on small changes in the local atomic structure, we would expect a rather broad continuum of states for a highly random, amorphous material. Experimental studies on nanocarbon materials often document excitation-wavelength-dependent luminescence. This behavior is usually attributed to surface trap states and/or broad particle size distributions. ${ }^{1,3,16}$ Our calculations suggest an alternative interpretation of this phenomenon; that the dispersion in the observed electronic and optical properties is caused by the diversity of underlying structures.

To quantify the effects of heteroatom-doping on the electronic properties of amorphous carbon will require an extensive study of the structure and dynamics of these materials based on larger unit cells. While this goes beyond the scope of the current work, the structures provided herein should provide a useful starting point for such investigations. 
Amorphous Carbon Nanodots: Nanodots were constructed by cleaving spherical particles of five different diameters between 1 and $2 \mathrm{~nm}$ from the periodic structures discussed above. All cleaved bonds were saturated with hydrogen and the geometries were optimized. All 25 final geometries (and additional calculation results) are provided in the form of EMPIRE arc files in the supporting information (SI_structures.zip). The electronic densities of states (DoS) for dots of the same size but based on different periodic structures are qualitatively similar (see Supporting Information, Fig. S3). We will therefore focus on dots based on a single structure (\#105) in the following discussion.

In Fig. 4, the electronic density of states of 1-2 nm nanodots is shown. The band gap decreases with increasing size. This effect is reminiscent to the size-confinement observed for inorganic quantum dots. ${ }^{37}$ However, additional individual isolated states appear in the band gap. 

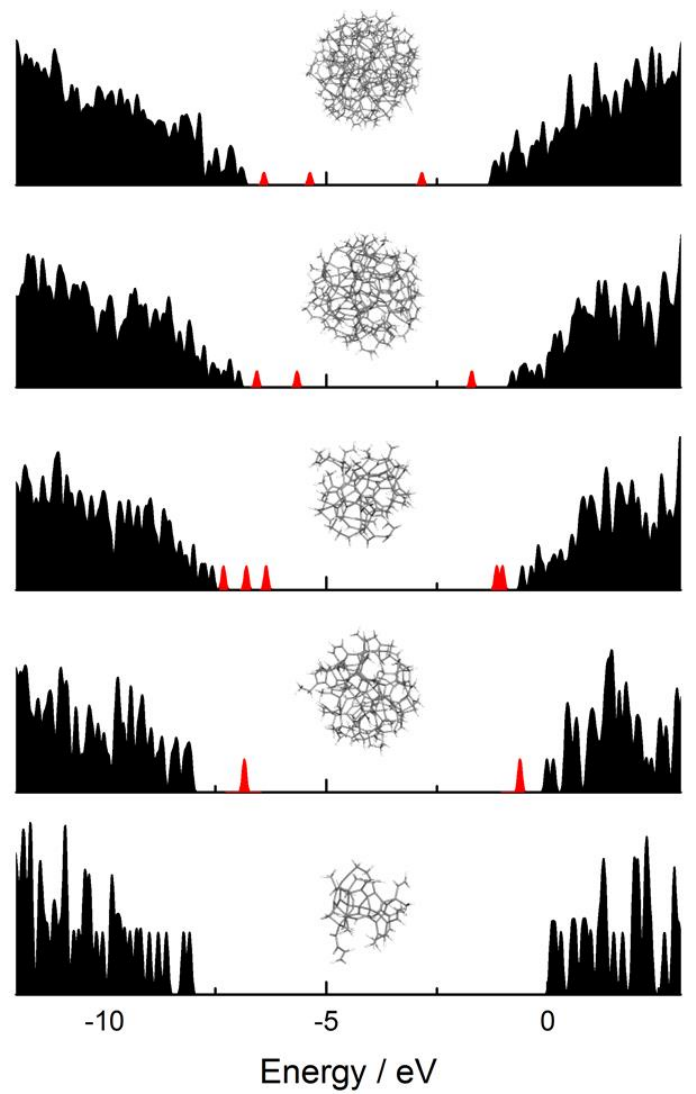

Figure 4. Diagrams of the electronic density of states for amorphous carbon nanodots, with diameters ranging from 1 (bottom) to $2 \mathrm{~nm}$ (top). Individual surface states are shown in red.

Surfaces of semiconducting materials often display localized electronic states in the band gap. This is caused by sudden changes of potentials from the periodic crystal structure to the environment, which occurs at surfaces, and may be avoided by passivation or crystal shape engineering. The DoS of the $2 \mathrm{~nm}$ dot, with molecular orbital isodensity plots of representative occupied (orange) and unoccupied (green) states as insets is shown in detail in Fig. 5. States in the continuous band region are relatively delocalized, while the mid-gap states are localized on the surface of the dots. 


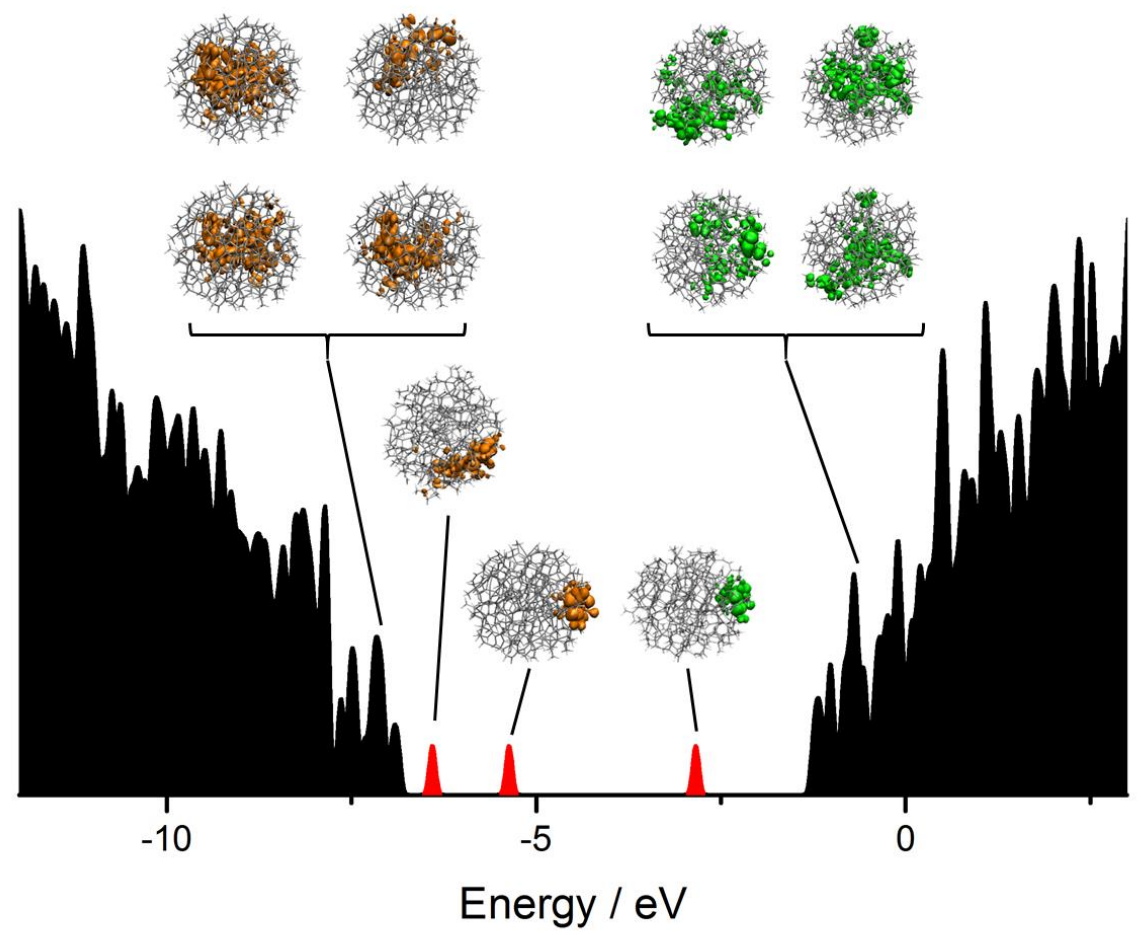

Figure 5. Diagram of the electronic density of states of a $2 \mathrm{~nm}$ amorphous carbon nanodot, and electron isodensity $\left(0.01 \mathrm{e}^{-} \AA^{-3}\right)$ plots of the orbitals corresponding to different band-like and surface states. Occupied orbitals are shown in orange, unoccupied ones in green.

Such states emerge because surface atoms are geometrically less constrained and, in turn, $\mathrm{sp}^{2}$ atoms at the surface form planar geometries more easily. Not surprising, the geometry near the particle surface is quite different from that in the bulk. A more detailed depiction of molecular orbitals that correspond to band-like and surface states is shown in in Fig. 6. 

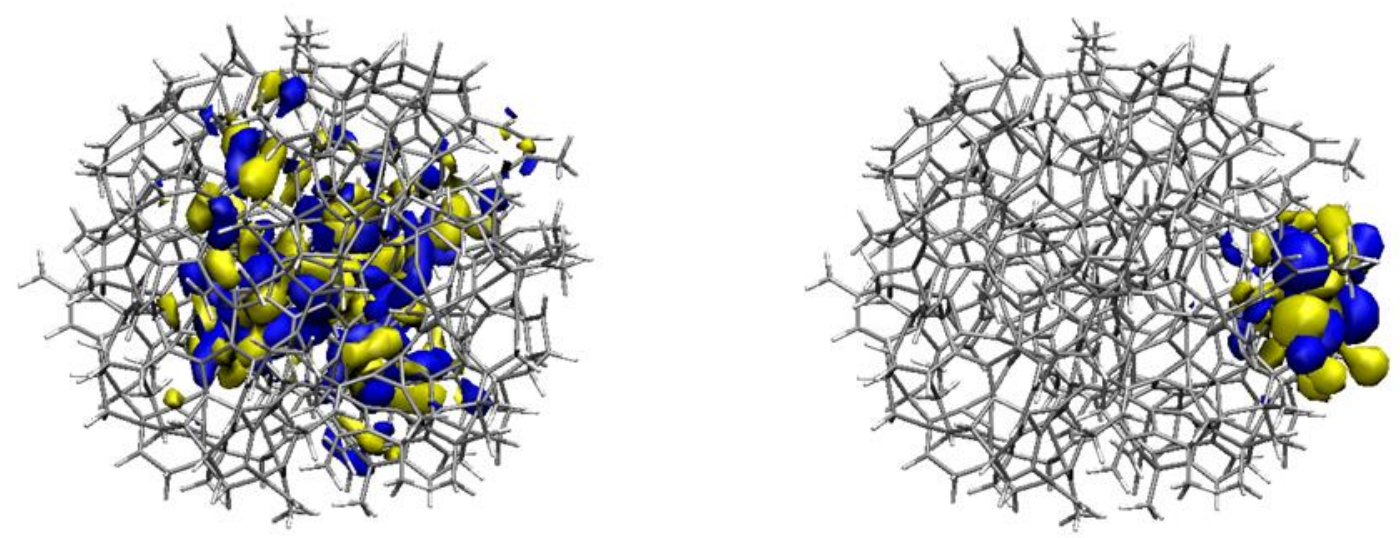

Figure 6. Exemplary molecular orbitals (isodensity surfaces of $0.01 \mathrm{e}^{-} \AA^{-3}$ ) corresponding to bandlike (left) and surface states (right) of a $2 \mathrm{~nm}$ nanodot.

An interesting consequence of these differences is an inhomogeneous charge distribution on the particle surface. Fig. 7 shows plots of different electronic properties on electron density isosurfaces. The molecular electrostatic potential (MEP) reveals patches of high negative (blue) and positive (red) MEP on the particle surface. These patches also possess characteristic reactivity, as shown in the local electron affinity $\left(\mathrm{EA}_{\mathrm{L}}\right)^{38,39}$ and ionization energy $\left(\mathrm{IE}_{\mathrm{L}}\right)^{40}$ maps. These two local properties have been used extensively to visualize ${ }^{41}$ or identify ${ }^{42,43}$ charge-transfer paths in molecules, crystals and devices and, more recently as an external potential for charge-transport calculations, ${ }^{44}$ in addition to their original uses as reactivity indicators. ${ }^{38-40}$ High $\mathrm{EA}_{\mathrm{L}}$ (red) indicates Lewis acidic, while low $\mathrm{IE}_{\mathrm{L}}$ (blue) goes hand in hand with Lewis basic properties. This is similar to colloidal quantum dots, which display facet-dependent electronic properties. ${ }^{45-47}$ 
(a)

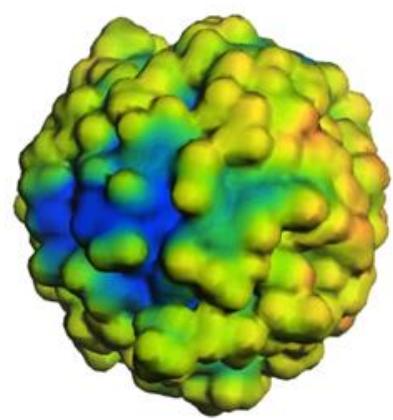

(b)

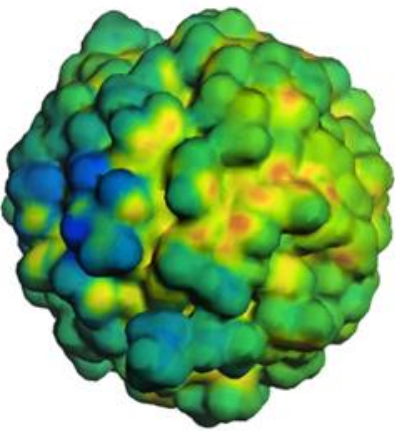

(c)

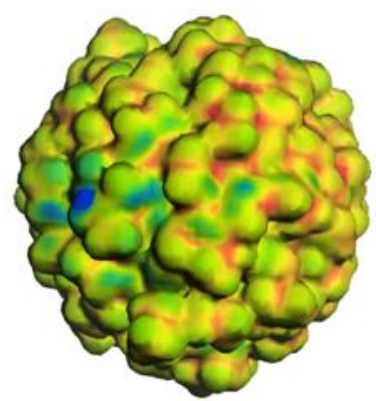

Figure 7. Maps of different properties on electron isodensity surfaces $\left(0.03 \mathrm{e}^{-} \AA^{-3}\right)$ of a $2 \mathrm{~nm}$, nanodot. (a) Plot of MEP from -50 (blue) to $50 \mathrm{kcal} \mathrm{mol}^{-1}$ (red). (b) Plot of EAL from -150 (blue) to $5 \mathrm{kcal} \mathrm{mol}^{-1}$ (red). (c) Plot of IEL from 270 (blue) to $500 \mathrm{kcal} \mathrm{mol}^{-1}$ (red).

For a quantitative analysis of the relationship between dot size and electronic structure, we considered the average electronic energy gaps of differently sized dots based on all five amorphous structures. Here, we considered three different energy gaps. We can determine both the actual band gap $\left(\Delta \mathrm{E}_{\mathrm{g}}\right)$ of the particles (ignoring surface states), and the HOMO-LUMO gap ( $\Delta \mathrm{E}_{\text {HOMO-LUMO) }}$ from the DoS. The latter is in most cases a gap between surface states. To obtain a more direct estimate the electronic band gap than that given by the eigenvalues of the frontier orbitals, we performed single point calculations on one-electron oxidized and reduced CNDs at the geometries of the neutral dots $\left(\Delta \mathrm{E}_{\mathrm{EA}-\mathrm{IP}}\right)$. The results are collected in Table 1 and show a monotonic decrease of $\Delta \mathrm{E}_{\mathrm{g}}$ and $\Delta \mathrm{E}_{\mathrm{HOMO}}$-LUMO with increasing particle size. Please note that the standard deviation of

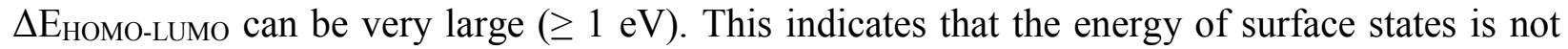
solely determined by the particle size, but also by the structure of the surface. The more physically justifiable values obtained for $\Delta \mathrm{E}_{\mathrm{EA}-\mathrm{IP}}$ show the same trend as $\Delta \mathrm{E}_{\mathrm{HOMO}-\mathrm{LUMO}}$. For small particles, 
the orbital relaxation effects included in $\Delta \mathrm{E}_{\mathrm{EA}-\mathrm{IP}}$ lead to $\sim 1 \mathrm{eV}$ smaller electronic gaps than in the frontier orbital model. For large particles the HOMO-LUMO gap is a good estimate.

Table 1. Average electronic gaps and standard deviations of amorphous carbon nanodots as a function of diameter.

\begin{tabular}{rrrr} 
Diameter $[\mathbf{n m}]$ & $\boldsymbol{\Delta} \mathbf{E}_{\mathbf{g}}[\mathbf{e V}]$ & $\boldsymbol{\Delta} \mathbf{E}_{\text {номо-Lumo }[\mathbf{e V}]}$ & $\boldsymbol{\Delta} \mathbf{E}_{\text {EA-IP }}[\mathbf{e V}]$ \\
\hline 1.0 & $8.46 \pm 0.3$ & $7.4 \pm 0.46$ & $6.15 \pm 0.32$ \\
1.25 & $7.74 \pm 0.46$ & $6.12 \pm 0.39$ & $5.06 \pm 0.6$ \\
1.5 & $6.87 \pm 0.19$ & $5.20 \pm 1.55$ & $4.51 \pm 0.88$ \\
1.75 & $6.03 \pm 0.28$ & $3.95 \pm 0.99$ & $4.02 \pm 1.2$ \\
2.0 & $5.71 \pm 0.22$ & $3.10 \pm 0.96$ & $3.38 \pm 0.31$
\end{tabular}

Finally, we turn to a description of the optical properties of the nanodots with different theoretical approaches (see the methods section for details). As an estimate of the optical bandgap ( $\mathrm{E}_{\mathrm{opt}}$ ), we considered the excitation energy to the lowest energy singlet excited state. The semiempirical methods INDO/S and AM1-CIS were applied to the entire size range of nanodots from 1-2 nm and all five structures. All molecular orbitals were included in the active space of the CIS calculations. The average excitation energies and corresponding standard deviations are shown in Fig. 8. Both methods predict a size dependence analogous to the results for the electronic bandgap. The AM1CIS excitation energies are consistently higher than the INDO/S ones. Either way, the predicted optical bandgaps of all dots lie in the optical or infrared part of the spectrum. The size dependency is strongest for small particles in the range of 1-1.5 $\mathrm{nm}$ and levels off afterwards. 


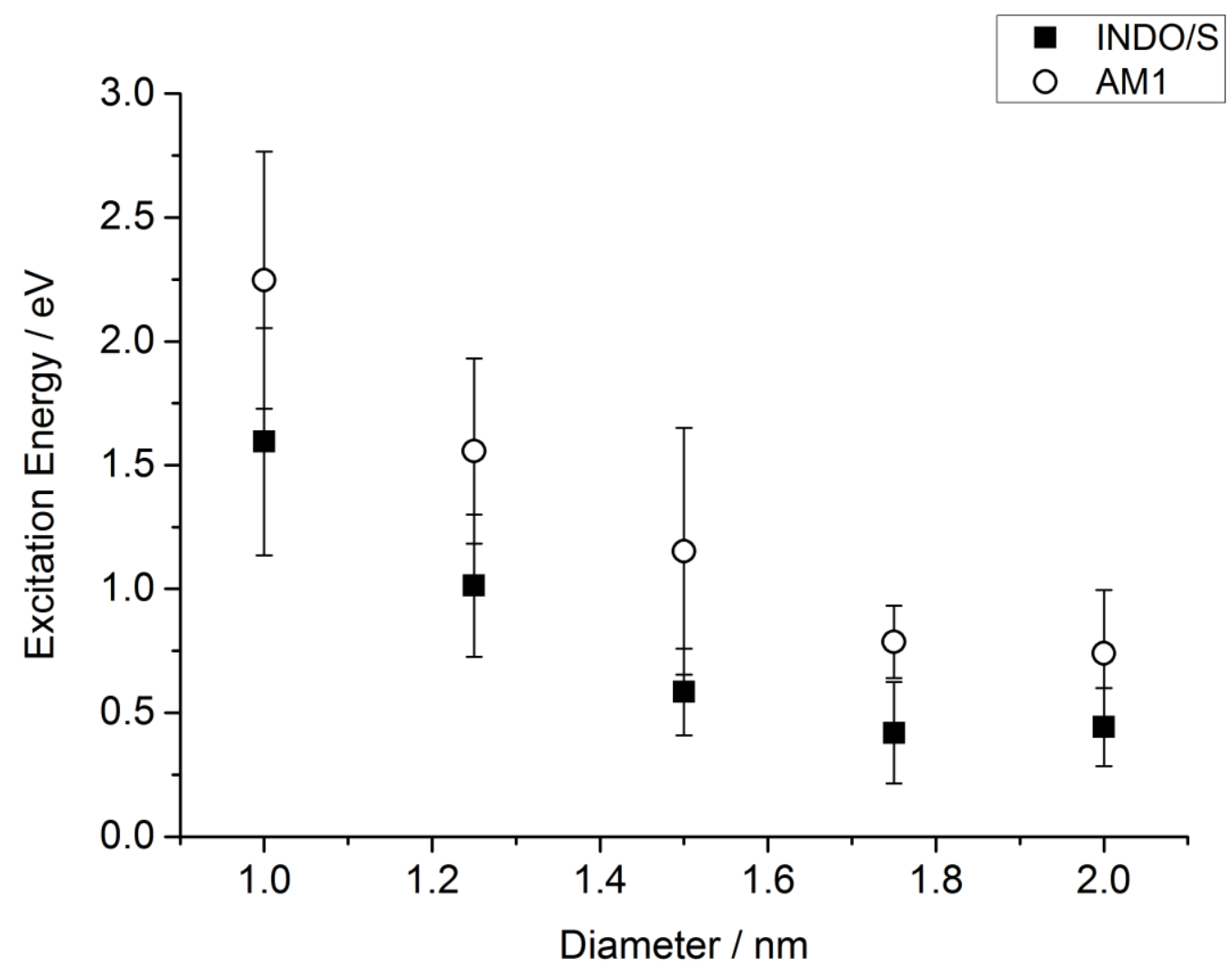

Figure 8. Average excitation energies of differently sized nanodots, calculated with INDO/S and AM1-CIS.

To assess the accuracy of these methods (which tend to underestimate vertical excitation energies), ${ }^{48}$ we compared them with time-dependent density-functional theory (TD-DFT) and AM1-UNO-CI ${ }^{23}$ for three nanodots sized between 1.0 and $1.5 \mathrm{~nm}$ (Figure 9.). Here we used the O-LYP functional with and without long range corrections and the 6-31G(d) basis set. ${ }^{28-31}$ As expected, there is a large discrepancy between the regular and long-range corrected DFT results. Excitation energies predicted by the former are even lower than with INDO/S, while the latter predicts higher values than AM1-CIS. This is due to the well-known tendency of DFT to 
underestimate the energy of charge transfer excitations, which is alleviated by the long range correction.
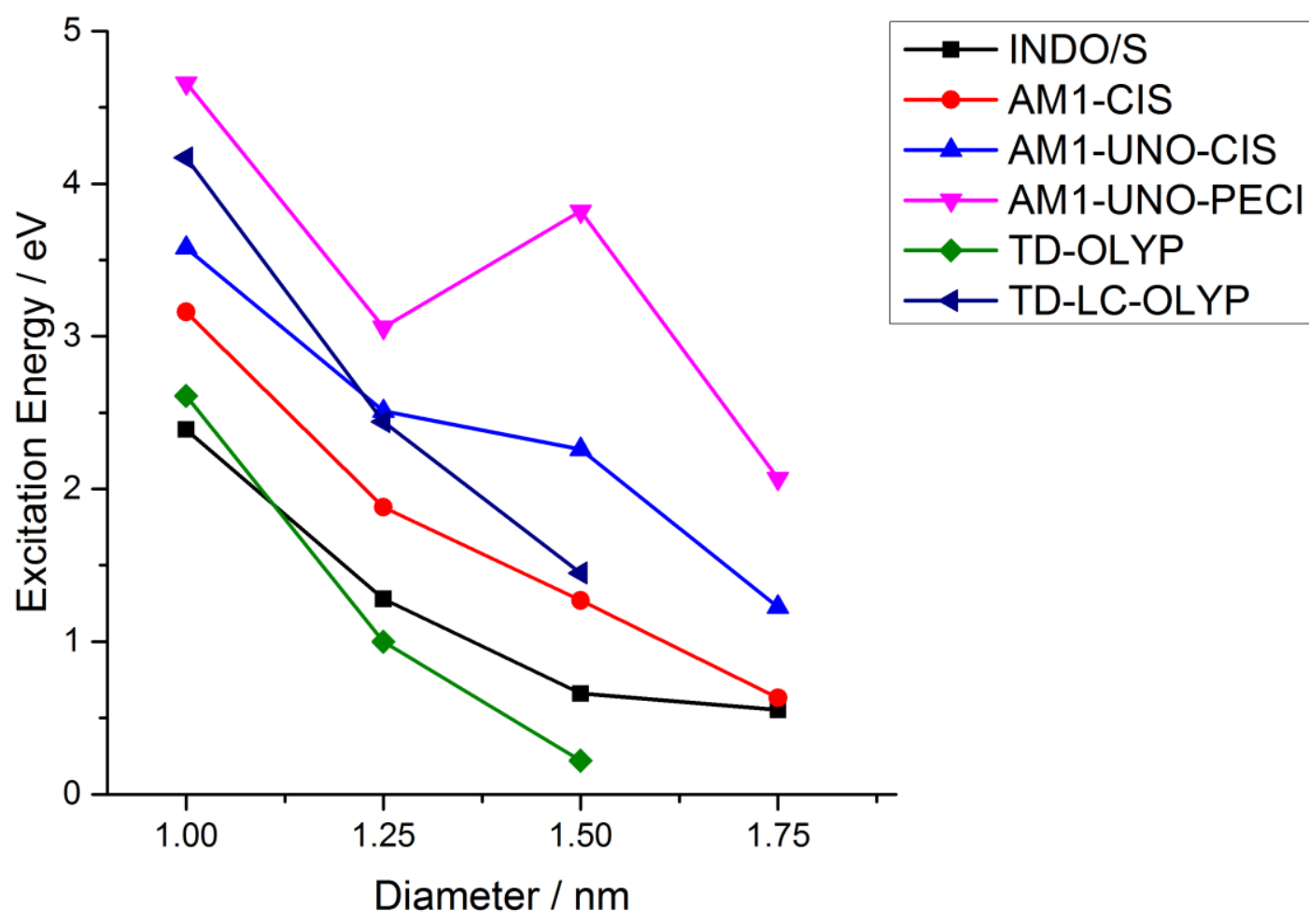

Figure 9. Excitation energies of differently sized nanodots based on structure \#105, calculated with different methods.

We conclude that the real optical band-gaps of the amorphous nanodots studied here may be up to $1 \mathrm{eV}$ higher than predicted by AM1-CIS. These conclusions are supported by the AM1-UNO-CI calculations, which have been found to give reliable band gaps at low cost. ${ }^{23}$ The UNO-CIScalculated band gaps are comparable to those given by TD-LC-OLYP but AM1-UNO-CIS overestimates the stability of triplet states relative to the ground state. This tendency can be corrected using AM1-UNO-PECI calculations,${ }^{49}$ which gives higher optical band gaps than TDLC-OLYP by about $0.5 \mathrm{eV}$. The trend towards lower band gaps for larger particles is found 
consistently with all configuration-interaction and TD-DFT calculations. AM1-UNO-PECI stands alone in predicting an increase in band gap for the $1.5 \mathrm{~nm}$ CND compared to that with $1.25 \mathrm{~nm}$ diameter.

These results illustrate that the prediction of optical band-gaps for these materials is difficult, because it is known that the available methods differ quite strongly. As mentioned above, the canonical semiempirical CIS methods tend to underestimate band-gaps. The same is true for charge-transfer excitations in TD-DFT without long-range corrections. The correlation effects for the ground state that are introduced by pair excitations in UNO-PECI are not size-consistent, leading to the observed deviation from the other methods in terms of size dependence. Therefore, we believe that the UNO-CIS and LC-TD-DFT results (which are in good agreement with each other) represent the best theoretical estimates for the bandgaps of these materials.

The trend of decreasing band gaps with increasing size has previously been shown for ordered $\mathrm{sp}^{2}-$ systems such as polycyclic aromatic hydrocarbons, ${ }^{50}$ graphene nanoribbons ${ }^{51}$ and nanoflakes ${ }^{52}$. Notably, it also holds for intrinsically disordered systems like the amorphous CNDs discussed here. Finally, note that the oscillator strengths of the $S^{0}-S^{1}$ transitions discussed here are typically less than 0.1 . This indicates that these states will mainly play a role in photoluminescence, while the absorption in the visible part of the spectrum will be fairly weak.

\section{Conclusion}

We have investigated the electronic properties of amorphous carbon nanomaterials. Their electronic structure depends most strongly on the geometry and less so on the atomic hybridization and heteroatom contents. Doping with heteroatoms can only work if the dopants are incorporated in a systematic manner. A truly random structure is expected to have a broad distribution of 
electronic states (even if the elemental composition is homogeneous throughout the material). Structures synthesized from molecular precursors are likely to have preferred geometrical features. In this case, a more homogeneous electronic structure can be expected.

When nanodots are formed from these structures, two additional factors come into play: Firstly, sub-band gap surface states arise. This allows optical transitions in the near-UV and visible range, and leads to positively and negatively charged sites at the nanodot surface. Secondly, the electronic structure is also influenced by the particle size. This is reflected in a size-confinement effect, especially evident for very small particles $(\mathrm{d}<1.5 \mathrm{~nm})$. Amorphous carbon nanodots surface features will dominate the behavior of nanodots in catalytic or electron-transfer applications.

\section{ASSOCIATED CONTENT}

Supporting Information. Plot of atom hybridization versus density (Fig. S1), AM1 lattice constants for diamond and graphite (Fig. S2), convergence of energy and band gap with periodic supercell size (Fig. S3), calculation results for all periodic structures (Table S1) and DoS plots for differently sized nanodots based on all $\mathrm{C}_{128}$ structures (Fig. S4). The archive SI_structures.zip contains the structures of 68 pure and doped periodic amorphous carbon phases in .cif format and 25 nanoparticles in the EMPIRE .arc format. Structure designations in the .zip file correspond to the ones in Table S1.

\section{AUTHOR INFORMATION}

\section{Corresponding Author}

*DMG: dirk.guldi@fau.de, TC: tim.clark@fau.de

\section{ACKNOWLEDGMENT}


This work was supported by the Deutsche Forschungsgemeinschaft as part of the Excellence Cluster "Engineering of Advanced Materials" and the Collaborative Research Centre SFB 953 "Synthetic Carbon Allotropes". The Bayerische Staatsregierung is also acknowledged for funding granted as part of the "Solar Technologies go Hybrid" initiative. Johannes T. Margraf is supported by a Beilstein Foundation Scholarship. Volker Strauss is supported by the "Universität Bayern e.V."

\section{REFERENCES}

1. Li, H.; Kang, Z.; Liu, Y.; Lee, S.-T. Carbon Nanodots: Synthesis, Properties and Applications. J. Mater. Chem. 2012, 22, 24230-24253.

2. Shen, J.; Zhu, Y.; Yang, X.; Li, C. Graphene Quantum Dots: Emergent Nanolights for Bioimaging, Sensors, Catalysis and Photovoltaic Devices. Chem. Commun. 2012, 48, 3686-3699.

3. Strauss, V.; Margraf, J. T.; Dolle, C.; Butz, B.; Nacken, T. J.; Walter, J.; Bauer, W.; Peukert, W.; Spiecker, E.; Clark, T.; Guldi, D. M. Carbon Nanodots - Towards a Comprehensive Understanding of their Photoluminescence. J. Am. Chem. Soc. 2014, 136, 17308-17316.

4. Yamijala, S. S.R.K.C.; Bandyopadhyay, A.; Pati, S. K. Electronic Properties of Zigzag, Armchair and their Hybrid Quantum Dots of Graphene and Boron-Nitride with and without Substitution: A DFT Study. Chem. Phys. Lett. 2014, 603, 28-32.

5. Zhu, B.; Sun, S.; Wang, Y.; Deng, S.; Qian, G.; Wang, M.; Hu, A. Preparation of Carbon Nanodots from Single Chain Polymeric Nanoparticles and Theoretical Investigation of The Photoluminescence mechanism. J. Mater. Chem. C 2013, 1, 580-586. 
6. Liu, H.; Lee, J. Y. Electric Field Effects on the Adsorption of CO on a Graphene Nanodot and the Healing Mechanism of a Vacancy in a Graphene Nanodot. J. Phys. Chem. C 2012, 116, 30343041.

7. Tang, J.; Zhang, Y.; Kong, B.; Wang, Y.; Da, P.; Li, J.; Elzatahry, A. A.; Zhao, D.; Gong, X.; Zheng, G. Solar-Driven Photoelectrochemical Probing of Nanodot/Nanowire/Cell Interface. Nano Lett. 2014, 14, 2702-2708.

8. Zarenia, M.; Chaves, A.; Farias, G. A.; Peeters, F. M. Energy Levels of Triangular and Hexagonal Graphene Quantum Dots: A Comparative Study Between the Tight-Binding and Dirac Equation Approach. Phys. Rev. B 2011, 84, 245403/1-12.

9. Zhang, M.; Bai, L.; Shang, W.; Xie, W.; Ma, H.; Fu, Y.; Fang, D.; Sun, H.; Fan, L.; Han, M.; Liub, C.; Yang, S. Facile Synthesis of Water-Soluble, Highly Fluorescent Graphene Quantum Dots as a Robust Biological Label for Stem Cells. J. Mater. Chem. 2012, 22, 7461-7467.

10. Saidi, W. A. Oxygen Reduction Electrocatalysis Using N-Doped Graphene Quantum-Dots. J. Phys. Chem. Lett. 2013, 4, 4160-4165.

11. Kumar, G. S.; Roy, R.; Sen, D.; Ghorai, U. K.; Thapa, R.; Mazumder, N.; Sahab, S.; Chattopadhyay, K. K. Amino-Functionalized Graphene Quantum Dots: Origin of Tunable Heterogeneous Photoluminescence. Nanoscale 2014, 6, 3384-3391.

12. Zhao, M.; Yang, F.; Xue, Y.; Xiao, D.; Guo, Y. A Time-Dependent DFT Study of the Absorption and Fluorescence Properties of Graphene Quantum Dots. ChemPhysChem 2014, 15, 950-957. 
13. Sk, M. A.; Ananthanarayanan, A.; Huang, L.; Lim, K. H.; Chen, P. Revealing the Tunable Photoluminescence Properties of Graphene Quantum Dots. J. Mater. Chem. C. 2014, 2, 69546960.

14. Santos, R. B. d.; Mota, F. d. B.; Rivelino, R. A Theoretical Evaluation of the Effect of Water on the Electronic Properties of Low Density Amorphous Carbon Nanoparticles. Carbon 2012, 50, 2788-2795.

15. Khavryuchenko, O. V.; Khavryuchenko, V. D.; Peslherbe, G. H. Density Functional Theory versus Complete Active Space Self-Consistent Field Investigation of the Half-Metallic Character of Graphite-Like and Amorphous Carbon Nanoparticles. J. Phys. Chem. A 2014, 118, 7052-7057.

16. Zhu, S.; Meng, Q.; Wang, L.; Zhang, J.; Song, Y.; Jin, H.; Zhang, K.; Sun, H.; Wang, H.; Yang, B. Highly Photoluminescent Carbon Dots for Multicolor Patterning, Sensors, and Bioimaging. Angew. Chem. Int. Ed. 2013, 52, 3953-3957.

17. Hennemann, M.; Clark, T. EMPIRE: A Highly Parallel Semiempirical Molecular Orbital Program: 1: Self-Consistent Field Calculations. J. Mol. Model. 2014, 20, 2331-2341.

18. Perkins, P. G.; Stewart, J. J. P. Cluster Model for Solids. J. C. S. Faraday II 1980, 76, 520533.

19. Stewart, J. J. P. A Practical Method for Modeling Solids Using Semiempirical Methods. J. Mol. Struct. 2000, 556, 59-67.

20. Stewart, J. J. P. Application of the PM6 Method to Modeling the Solid State. J. Mol. Model., 2008, $14,499-535$. 
21. Stewart, J. J. P. Optimization of Parameters for Semiempirical Methods V: Modification of NDDO Approximations and Application to 70 elements. J. Mol. Model., 2007, 13, 1173-1213.

22. Chen, Z:; Thiel, W. Performance of Semiempirical Methods in Fullerene Chemistry: Relative Energies and Nucleus-Independent Chemical Shifts. Chem Phys. Lett. 2003, 367, 15-25.

23. Dral. P. O.; Clark, T. Semiempirical UNO-CAS and UNO-CI: Method and Applications in Nanoelectronics. J. Phys. Chem. A 2011, 115, 11303-11312.

24. Marks, N.; Cooper, N.; McKenzie, D.; McCulloch, D.; Bath, P.; Russo, S. Comparison of Density-Functional, Tight-Binding, and Empirical Methods for the Simulation of Amorphous Carbon. Phys. Rev. B 2002, 65, 075411/1-9.

25. Li, L.; Xu, M.; Song, W.; Ovcharenko, A.; Zhang, G.; Jia, D. The Effect of Empirical Potential Functions on Modeling of Amorphous Carbon Using Molecular Dynamics Method. Appl. Surf. Sci. 2013, 286, 287-297.

26. Dewar, M. J. S.; Zoebisch, E. G.; Healy, E. F.; Stewart, J. J. P. Development and Use of Quantum Mechanical Molecular Models. 76. AM1: A New General Purpose Quantum Mechanical Molecular Model. J. Am. Chem. Soc. 1985, 107, 3902-3909.

27. Ridley, J. E.; Zerner, M. C. An Intermediate Neglect of Differential Overlap Technique for Spectroscopy: Pyrrole and the Azines. Theor. Chem. Acc. 1973, 32, 111-134.

28. Handy, N. C.; Cohen, A. J. Left-Right Correlation Energy. Mol. Phys. 2001, 99, 403-412.

29. Lee, C.; Yang, W.; Parr, R. G. Development of the Colle-Salvetti Correlation-Energy Formula Into a Functional of the Electron Density Phys. Rev. B 1988, 37, 785-789. 
30. Iikura, H.; Tsuneda, T.; Yanai, T.; Hirao, K. Long-Range Correction Scheme for Generalized-Gradient-Approximation Exchange Functionals J. Chem. Phys. 2001, 115, 35403544.

31. Hariharan P. C.; Pople, J. A. Influence of Polarization Functions on Molecular-Orbital Hydrogenation Energies Theor. Chem. Acc. 1973, 28, 213-222.

32. Frisch, M. J.; Trucks, G. W.; Schlegel, H. B.; Scuseria, G. E.; Robb, M. A.; Cheeseman, J. R.; Scalmani, G.; Barone, V.; Mennucci, B.; Petersson, G. A. et. al. Gaussian 09, Revision C.01, Gaussian, Inc., Wallingford CT, 2009.

33. Clark, T.; Alex, A.; Beck, B.; Burkhardt, F.; Chandrasekhar, J.; Gedeck, P.; Horn, A.; Hutter, M.; Martin, B.; Dral, P. O et. al. VAMP 11.0, Erlangen, 2011.

34. Dral, P. O.; Kivala, M.; Clark, T. Doped Polycyclic Aromatic Hydrocarbons as Building Blocks for Nanoelectronics: A Theoretical Study. J. Org. Chem., 2013, 78, 1894-1902.

35. Jones, A. P. Heteroatom-doped hydrogenated amorphous carbons, a-C:H:X - "Volatile" Silicon, Sulphur and Nitrogen Depletion, Blue Photoluminescence, Diffuse Interstellar Bands and Ferromagnetic Carbon Grain Connections. $A \& A$ 2013, 555, A39.

36. Adamopoulos, G.; Godet, C.; Zorba, T.; Paraskevopoulos, K. M.; Ballutaud, D. Electron Cyclotron Resonance Deposition, Structure, and Properties of Oxygen Incorporated Hydrogenated Diamondlike Amorphous Carbon Films. J. Appl. Phys. 2004, 96, 5456-5461. 
37. Murray, C. B.; Norris, D. J.; Bawendi, M. G. Synthesis and Characterization of Nearly Monodisperse CdE (E = Sulfur, Selenium, Tellurium) Semiconductor Nanocrystallites. J. Am. Chem. Soc. 1993, 115, 8706-8715.

38. Ehresmann, B.; Martin, B.; Horn, A. H. C.; Clark, T. Local Molecular Properties and Their use in Predicting Reactivity. J. Mol. Model. 2003, 9, 342-347.

39. Clark, T. The Local Electron Affinity for Non-Minimal Basis Sets. J. Mol. Model. 2010, 16, $1231-1238$.

40. Sjoberg, P.; Murray, J. S.; Brinck, T.; Politzer, P. Average Local Ionization Energies on the Molecular-Surfaces of Aromatic Systems as Guides to Chemical-Reactivity. Can. J. Chem. 1990, $68,1440-1443$.

41. Atienza, C.; Martin, N.; Wielopolski, M.; Haworth, N.; Clark, T.; Guldi, D. M. Tuning Electron Transfer Through $p$-Phenyleneethylene Molecular Wires. Chem. Commun., 2006, $3202-$ 3204.

42. Jäger, C. M.; Schmaltz, T.; Novak, M.; Khassanov, A.; Vorobiev, A.; Hennamann, M.; Krause, A.; Dietrich, H.; Zahn, D.; Hirsch, A.; Halik, M.; Clark, T. Improving the Charge Transport in Self-assembled Monolayer Field-effect Transistors - From Theory to Devices. J. Am. Chem. Soc., 2013, 135, 4893-4900.

43. Etschel, S.; Waterloo, A.; Margraf, J. T.; Amin, A. Y.; Hampel, F.; Jäger, C. M.; Clark, T.; Halik, M., Tykwinski, R. R. An Unsymmetrical Pentacene Derivative with Ambipolar Behavior in Organic Thin-Film Transistors. Chem. Commun., 2013, 49, 6725-6727. 
44. Bauer, T. Multi-Agenten-Simulation Organischer Feldeffekttransistoren. Ph.D. Thesis, Friedrich-Alexander-Universität Erlangen-Nürnberg, 2014.

45. Margraf, J. T.; Ruland, A.; Sgobba, V.; Guldi, D. M.; Clark, T. Theoretical and Experimental Insights into the Surface Chemistry of Semiconductor Quantum Dots. Langmuir 2013, 29, 1545015456.

46. Voznyy, O.; Thon, S. M.; Ip, A. H.; Sargent, E. H. Dynamic Trap Formation and Elimination in Colloidal Quantum Dots. J. Phys. Chem. Lett. 2013, 4, 987-992.

47. Voznyy, O.; Sargent, E. H. Atomistic Model of Fluorescence Intermittency of Colloidal Quantum Dots. Phys. Rev. Lett. 2014, 112, 157401/1-5.

48. Silva-Junior, M. R.; Thiel, W. Benchmark of Electronically Excited States for Semiempirical Methods: MNDO, AM1, PM3, OM1, OM2, OM3, INDO/S, and INDO/S2. J. Chem. Theory Comput. 2010, 6, 1546-1564.

49. Clark, T.; Chandrasekhar J. NDDO-Based CI Methods for the Prediction of Electronic Spectra and Sum-Over-States Molecular Hyperpolarizabilities, Israel J. Chem. 1993, 33, 435448.

50. Malloci G.; Cappellini, G.; Mulas, G.; Mattoni, A. Electronic and Optical Properties of Families of Polycyclic Aromatic Hydrocarbons: A Systematic (Time-Dependent) Density Functional Theory Study Chem. Phys. 2011 384, 19-27.

51. Son, Y.-W.; Cohen, M. L., Louie S. G. Energy Gaps in Graphene Nanoribbons Phys. Rev. Lett. 2006, 97, 216803. 
52. Cocchi, C.; Ruini, A.; Prezzi, D.; Caldas, M. J.; Molinari, E. Designing All-Graphene Nanojunctions by Covalent Functionalization J. Phys. Chem. C 2011, 115, 2969-2973. 
Table of Contents Graphic:
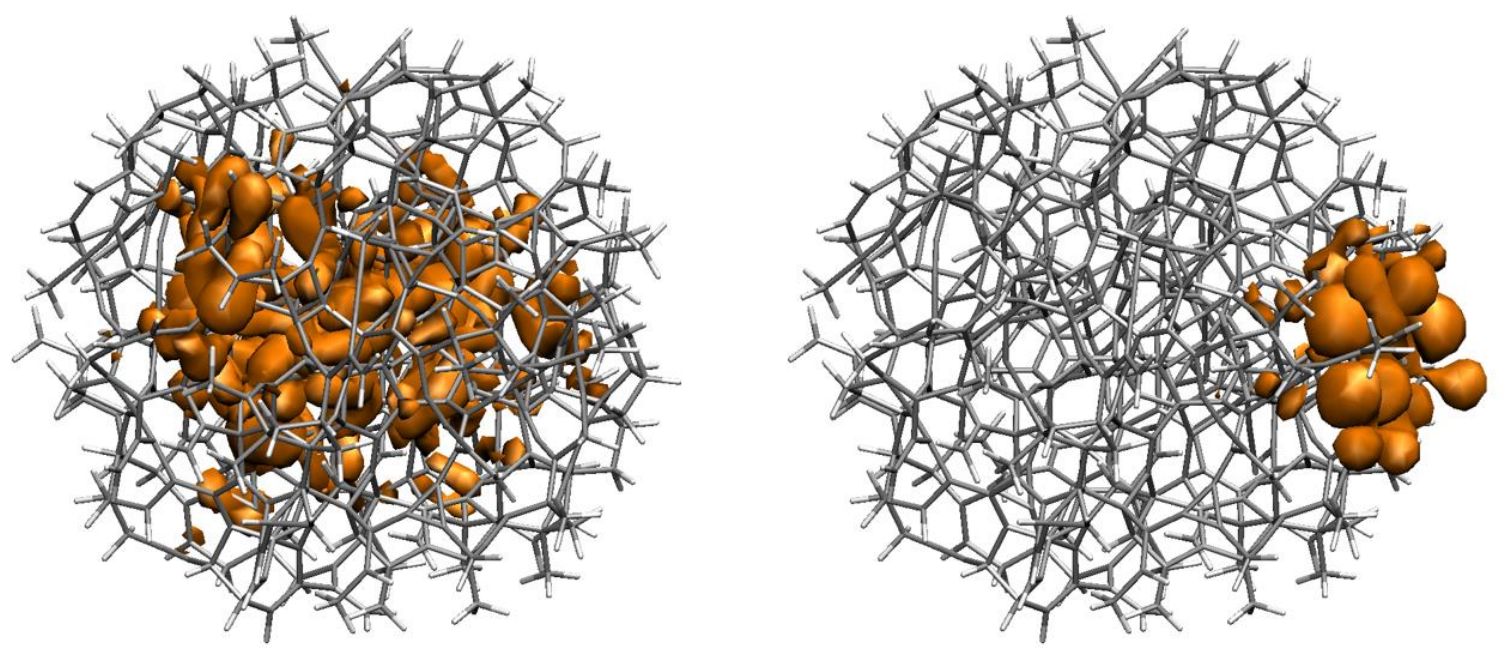